\title{
La fabrique de l'immobilité. Réflexion critique à partir de travaux d'enquête menés à Dakar (Sénégal)
}

David Lessault

\section{CpenEdition}

Journals

Édition électronique

URL : https://journals.openedition.org/e-migrinter/223

DOI : 10.4000/e-migrinter.223

ISSN : 1961-9685

Éditeur

UMR 7301 - Migrinter

\section{Édition imprimée}

Date de publication : 1 septembre 2013

Pagination : 21-27

ISSN : 1961-9685

\section{Référence électronique}

David Lessault, "La fabrique de l'immobilité. Réflexion critique à partir de travaux d'enquête menés à Dakar (Sénégal) », e-Migrinter [En ligne], 11 | 2013, mis en ligne le, consulté le 20 mai 2021. URL http://journals.openedition.org/e-migrinter/223 ; DOI : https://doi.org/10.4000/e-migrinter.223 


\section{e-migrinter) \\ La fabrique de l'immobilité Réflexion critique à partir de travaux d'enquête menés à Dakar (Sénégal)}

David Lessault

D

ans le cadre de l'introduction à ces journées, je propose une réflexion critique menée à partir de mon expérience de recherche : une série de travaux conduits sur Dakar qui s'appuient pour l'essentiel sur des programmes d'enquêtes sociodémographiques réalisés au Sénégal.
Cette contribution s'articule autour de deux questions centrales : dans un premier temps, comment la question des immobiles ou de l'immobilité émerge de façon implicite dans mes travaux sur la mobilité sénégalaise ? Et dans un second temps, en quoi l'étude des mobilités ou immobilités est conditionnée a priori par l'usage de catégories de collecte et d'analyse rencontrées dans les enquêtes sur lesquelles j’ai travaillées?

Pour répondre à la première question, je propose une relecture rapide de mes travaux qui mettent en évidence des situations d'immobilité dans le contexte dakarois. Pour alimenter la seconde question, je pose un regard critique sur les catégories utilisées dans les enquêtes sur les migrations au Sénégal pour qualifier la circulation, pour rendre compte de deux types d'états (mobiles/immobiles), et finalement pour envisager les rapports entre individus mobiles et individus immobiles.

\section{Des travaux centrés sur la mobilité...}

Mes recherches privilégient une lecture des mobilités sénégalaises en lien avec les dynamiques socio-économiques (conditions d'habitat et accès au logement) et démo-spatiales (logiques du peuplement urbain et production urbaine en périphérie) de l'agglomération de Dakar. L'un des objectifs est d'étudier comment les mobilités spatiales considérées à différentes échelles de temps et d'espace contribuent aux transformations récentes de la ville.

La question de "l'immobilité » ne va donc pas de soi dans ma démarche qui exprime d'une certaine façon un parti pris, 
un a priori (un biais ?) : rechercher des clés d'explication au changement dans le mouvement et laisser de côté, au moins provisoirement, ce qui est statique, immobile dans une société ou au sein d'un espace. Dans l'approche développée, le regard est porté sur «le sens du mouvement», celui de la ville qui se transforme, celui des circulations par lesquelles elle se transforme. Pour autant, l'immobilité est directement en jeu au moment où «l'objet mobilité » est interrogé puisqu'elle lui est autant antinomique qu'indissociable.

\section{... aux références implicites à l'immobilité}

En effet, à la relecture de mes travaux, les références à l'immobilité ou les situations d'immobilité émergent, de manière assez évidente mais souvent implicite. Je peux en citer plusieurs exemples concrets : cherchant dans ma thèse à mieux comprendre la construction de l'espace périurbain à Dakar par des mobilités résidentielles à caractère centrifuge, j'observe finalement le rôle déterminant des populations locales primo-occupants - dans l'attribution des parcelles à construire ou des logements : l'espace périurbain apparait comme le produit de relations complexes entre «mobiles» («nouveaux arrivants», candidats à la propriété immobilière) et «immobiles» (autochtones des villages lebou, propriétaires coutumiers du sol). Le déménagement en périurbain, presque devenu une condition d'accès à la propriété à Dakar, traduit aussi une localisation résidentielle contraignante. J'ai parlé dans ma thèse " d'individus captifs » pour caractériser la situation d'habitants périurbains qui faute de ressources financières suffisantes et d'infrastructures de transports étaient alors «coupés» de la ville. Quand l'urbanisation précède la ville, la mobilité résidentielle peut conduire à l'immobilité quotidienne...
Examinant, dans mes travaux avec Alioune Diagne - alors démographe à l'IRD à Dakar - le calendrier de la sortie de l'hébergement des jeunes adultes à Dakar ${ }^{1}$, nous concluons au retard à la décohabitation chez les dernières générations. Les difficultés croissantes pour trouver un emploi stable et la situation du marché locatif limitent les possibilités d'accès à un logement autonome, mais le prolongement des cohabitations relève aussi de stratégies familiales pour mutualiser les ressources et éviter la dispersion des dépenses en temps de crise. Cette "immobilité résidentielle», à un moment particulier du cycle de vie généralement caractérisé par une forte mobilité résidentielle et que l'on pensait de prime abord «contrainte » ou «subie », est aussi collectivement souhaitée et négociée. Elle n'empêche d'ailleurs pas la réalisation des projets professionnels et matrimoniaux, elle peut même les faciliter. Quoi qu'il en soit, cette forme d'immobilité prolongée a des répercussions sur la composition des ménages à Dakar - dont la taille moyenne reste relativement élevée pour une ville ouest-africaine - et par conséquent sur la redistribution des pouvoirs au sein des familles.

$\mathrm{Au}$ cours de mon post-doctorat à l'Ined mes recherches se sont orientées sur les migrations internationales au Sénégal, dans le cadre de la mise en place du projet Migrations entre l'Afrique et l'Europe (MAFE) ${ }^{2}$. Nous avons observé des taux d'émigration internationale annuels finalement modérés alors que l'enquête révèle à Dakar une proportion importante de ménages concernés par la migration internationale (plus d'un ménage sur deux déclare qu'au moins un de ses «membres» réside actuellement à l'étranger). Au-delà de l'aspect cumulatif de la migration dans le temps, c'est la question de «ceux qui restent» qui est posée, l'enjeu de la circulation internationale étant davantage

\footnotetext{
${ }^{1}$ www.ceped.org/?Emancipation-residentielle

2 www.mafeproject.com
} 
situé au niveau du " groupe », à l'échelle de la famille : quel est l'effet du départ des uns sur le sort des autres? Par ailleurs, dans cette enquête, le rapport entre mobiles et immobiles est envisagé au niveau individuel par la comparaison de trois souspopulations : «migrants », " non migrants », «migrants de retour» pour désigner «ceux qui partent», "ceux qui restent» et «ceux qui rentrent ». Le choix de ces catégories de collecte et d'analyse "englobantes» a une implication directe sur la lecture des rapports mobilité/immobilité.

Enfin, dernier exemple, dans des travaux plus récents menés avec Christophe $\mathrm{Imbert}^{3}$, nous étudions à partir des données de l'enquête MAFE déjà évoquée, les trajectoires résidentielles de plus de mille habitants de l'agglomération de Dakar pour éclairer les logiques de peuplement de la ville. Deux faits marquants ressortent de nos analyses sur la mobilité résidentielle: i) la part importante d'individus dont l'intégralité de la trajectoire résidentielle se situe à l'intérieur de la ville et ii) la concentration des parcours dans certains secteurs résidentiels caractéristiques.

Ces observations sur la stabilité résidentielle des habitants de Dakar appellent une réflexion sur les nouvelles logiques du peuplement urbain et sur les catégories généralement utilisées pour définir la mobilité spatiale.

Ces divers constats incitent à une réflexion plus aboutie sur les situations d'immobilité et les manières de les caractériser. Les situations d'immobilité contraintes, de stabilité résidentielle, les sédentarités subies ou choisies qui caractérisent d'ailleurs les «états » les plus fréquents et les plus durables dans la vie d'un individu soulèvent d'importantes questions de société et interrogent autrement le rapport à l'espace. Elles méritent sans

\footnotetext{
${ }^{3}$ www.uclouvain.be/cps/ucl/doc/demo/docum ents/Lessault Imbert.pdf
}

doute un examen particulier et plus systématique. Mais surtout, la manière d'appréhender les rapports mobilité/immobilité et la façon d'en rendre compte dépendent de la construction et de l'usage des catégories. Au-delà de certaines « immobilités » constatées dans le contexte dakarois, ce sont les catégories d'analyse utilisées pour les observer que l'on peut mettre en question. Je propose de les interroger ici à partir de l'enquête MAFE à laquelle j’ai participé.

\section{Comment sont abordées « Immobilités » et circulation dans l'enquête MAFE - 2008 ?}

MAFE est un programme d'enquêtes spécialement conçu pour étudier les circulations internationales entre l'Afrique subsaharienne et l'Europe. Il s'est largement inspiré dans sa conception des enquêtes biographiques menées dans les grandes villes d'Afrique de l'Ouest (Travaux de Philippe Antoine) ou au Sénégal (EMUS - Enquête sur les Migrations et l'Urbanisation au Sénégal, Philippe Bocquier) et pour sa dimension inter - ou trans - nationale des travaux de Douglas Massey sur les migrations mexicaines.

Son objectif est de produire des données quantitatives permettant d'expliquer les migrations internationales, d'étudier les pratiques de circulation des Subsahariens et d'examiner l'effet de ces pratiques sur le développement des pays d'origine. Le rapport mobilité/immobilité est envisagé à deux niveaux :

- au niveau individuel par la comparaison des comportements des «migrants» et des «non migrants». Le migrant est considéré comme un individu ayant effectué au cours de sa vie au moins une migration internationale, c'est-à-dire un changement de pays où il a résidé pour une durée d'au moins un an. Par opposition, le « non migrant» est celui qui n'a jamais résidé à l'étranger pour une durée d'au moins un an au cours de sa vie; 
- au niveau du «groupe» le rapport mobiles/immobiles est appréhendé dans le cadre des relations entre le migrant/le circulant et l'entité socio-familiale à laquelle il est rattaché, avec un parti pris méthodologique important: privilégier l'échelle internationale des pratiques.

Deux hypothèses générales ont présidé à la mise en place du dispositif d'enquête :

(1) alors que les migrations internationales d'origine subsaharienne sont perçues comme un mouvement d'entrée à sens unique en Europe, elles reposeraient davantage sur des pratiques de va-et-vient entre pays de départ africains et pays de destination européens ;

(2) cette circulation migratoire et ses implications économiques (transferts d'argent et de biens, investissements économiques, initiatives des migrants «de retour ») contribueraient à améliorer sensiblement les conditions de vie des familles restées dans le pays d'origine, voire au-delà au développement économique et aux transformations des régions de départ.

Dans le cadre de la première hypothèse, c'est la question de l'appréhension de la migration en terme de circulation qui nous intéresse ici : de la prise en compte de sa nature réversible, de son aspect renouvelable, de sa temporalité, de son échelle spatiale d'observation. Comment la définition de ces différents critères conditionnent-ils la lecture des (im)mobilités ? Dans le cadre de la seconde hypothèse, c'est la question du rattachement de la migration à un groupe qui est soulevée afin d'identifier à qui profite la circulation internationale.

\section{Qui circule ? Qui est « immobile » ? Une question d'échelle}

Les analyses biographiques réalisées dans le cadre de ce projet accordent une attention exclusive aux trajectoires migratoires. Elles montrent des changements de pays dans lequel la personne a passé au moins un an de sa vie (migration internationale). La nature biographique des données est d'un intérêt qui n'est plus à démontrer dans l'étude des migrations : prise en compte du caractère renouvelable et réversible de la migration, mise en regard des calendriers migratoires, familiaux et professionnels, etc.

Ainsi, ces analyses ont permis la mise en évidence quantitative des pratiques de circulation migratoire des Sénégalais à l'échelle internationale: «un migrant sénégalais sur quatre [est] revenu dans son pays d'origine après dix ans passés à l'étranger» (Beauchemin, et al, MAFE) ${ }^{4}$. Elles ont aussi contribué à formuler et tester certaines hypothèses en termes d'entraves à la circulation migratoire liées aux politiques restrictives de l'immigration dans les pays du Nord, d'installation prolongée et d'ancrage plus systématique des migrants internationaux dans les pays d'accueil; soulignant également leur capacité à développer d'autres formes de circulation (transferts et investissements économiques, séjours courts en particulier) qui leur permettent de maintenir un lien fort avec le pays d'origine.

Toutefois, les catégories employées pour aborder la mobilité et l'immobilité apparaissent d'emblée très restrictives puisqu'elles tendent à réduire l'immobilité à la «non migration» et vice versa. La distinction opérée a priori entre « migrant » et «non-migrant» pour analyser les comportements sociaux, économiques et familiaux apparait limitée car trop englobante. Et c'est finalement préjuger d'un fort pouvoir explicatif de la «migration internationale » dans la destinée d'un individu et inversement, cantonner ceux qui ne migrent pas ou «qui restent» dans une situation "passive » en les privant du rôle d'acteurs qu'ils assurent à travers

\footnotetext{
${ }^{4}$ http://www.ined.fr/fichier/t telechargement/57140 /telechargement fichier_fr_wp7 flahaux etal 2010.p $\underline{\mathrm{df}}$
} 
d'autres pratiques spatiales exprimées à d'autres échelles.

C'est ce qui à mon sens pose problème dans les enquêtes sociodémographiques sur les villes africaines sur lesquelles j'ai travaillé (EMUS, 1993 ; JEREMI, 2001; MAFE, 2008). Dans ce cadre d'observation, la tentation est grande de réduire la circulation à une échelle spatiale (interne ou internationale) et temporelle (seuil d'un an) et donc de considérer comme «immobiles » tous ceux qui ne migrent pas.

Pourtant le recueil des biographies résidentielles, quand elle permet de localiser les différents logements occupés par un individu au cours de sa vie autorise une analyse plus fine en termes de trajectoires résidentielles et non plus simplement migratoires, ce qui permet de poser en d'autres termes les états de mobilité et d'immobilité résidentielle. C'est une question d'échelle de la mobilité et d'articulation des échelles de la mobilité qui est posée.

La prise en compte de toutes les localisations résidentielles occupées par un individu au cours de sa vie, y compris à l'échelle intra-urbaine, et selon des durées variables, s'avère nécessaire à une étude plus aboutie de la mobilité et à l'approche de l'immobilité résidentielle. $\mathrm{La}$ trajectoire résidentielle permet alors de lire une succession de périodes d'immobilité résidentielle entrecoupées de changements de résidence, s'ils ont lieu. Dans le temps, la trajectoire de la plupart des individus est dominée par les périodes de stabilité au sein de logements successifs. Et, dans cette optique, la localisation résidentielle apparait alors comme une variable importante pour mieux saisir des effets de contextes locaux et/ou identifier des espaces de « rétention résidentielle » ou des lieux d'ancrage au sein des espaces urbains en particulier.

Pour autant, l'approche en termes de trajectoire résidentielle ne résout pas tout et deux principales limites persistent dans cette appréhension des (im)mobilités :

- Comment saisir les périodes de multi-résidence et d'instabilité résidentielle (infra-annuelle)?

- Comment capter les formes de mobilité plus «temporaires»: mobilité quotidienne, déplacements pour loisirs, tourisme, affaires, visites familiales, etc ?

L'idée centrale proposée au débat est que la prise en compte de la complexité des mobilités individuelles exprimées à différentes échelles de temps et d'espace rend de plus en plus floue la frontière mobilité/immobilité, mobiles/immobiles, là où certaines catégories de collecte et d'analyse assez courantes ont tendance à la figer et à lui donner un fort pouvoir discriminant.

\section{À qui profite la circulation?}

La définition du ménage retenue dans l'enquête MAFE est assez classique: c'est " un groupe d'individus qui vivent ensemble et mettent en commun tout ou partie de leurs ressources pour subvenir à leurs besoins essentiels (logement et nourriture en particulier) »(Pilon et Vignikin, 2007). Pour être considérés comme membres du ménage, les individus doivent y résider pour une durée d'au moins six mois (révolus ou prévus).

L'originalité de l'enquête vient plutôt de l'intégration dans le questionnaire des migrants du ménage, c'est-à-dire d'individus qui, par définition, ne sont pas des membres du ménage (puisqu'ils vivent ailleurs) mais qui lui sont liés. Qui sont-ils? Le questionnaire ménage enregistre plusieurs catégories de migrants internationaux, c'està-dire de personnes résidant en dehors du Sénégal : les enfants du chef de ménage, le(s) conjoint(s) des membres du ménage, les personnes apparentées au chef de ménage ou à sa/son conjoint(e) qui ont eu des contacts réguliers (les enquêtés pouvaient interpréter librement cette notion de régularité des contacts, tant du point de vue 
du mode de relation - téléphone, visite, courrier, transferts financiers... - que de la périodicité) avec le ménage dans les douze mois précédant l'enquête.

Le projet MAFE a délibérément retenu une définition extensive des migrants du ménage afin de chercher à capter l'ensemble des personnes qui peuvent contribuer à l'économie domestique. Au total, suivant cette définition, il y a $53 \%$ de ménages avec migrant(s), c'est-à-dire déclarant des migrants actuels, dans la région de Dakar.

Partant de cette définition extensive lors de la collecte, certaines analyses ont cherché à affiner le rôle de la migration dans l'amélioration des conditions de vie des familles en distinguant différents types de ménages avec migrant(s) :

- selon le statut migratoire des individus: au-delà du rôle des migrants actuels (individus qui résident en dehors du Sénégal au moment de l'enquête), on étudie si les migrants de retour ont une influence spécifique sur les conditions de vie;

- selon le nombre de migrants : l'idée est de tester l'existence d'un potentiel effet cumulatif, selon lequel le potentiel d'investissement est plus fort à plusieurs, en particulier quand il concerne l'habitat qui nécessite d'importantes dépenses;

- selon la destination des migrants : on teste l'hypothèse selon laquelle les migrants installés en Europe, du fait de leurs revenus a priori supérieurs et du fait de pratiques d'habitat culturellement différentes, contribuent davantage à la transformation des logements de Dakar que leurs homologues installés en Afrique ;

- selon la nature des liens entre les migrants et le chef de ménage : on peut alors chercher à comprendre si les solidarités entre les migrants et les ménages dakarois s'exercent dans le cadre de liens familiaux étroits (conjoints et enfants du chef de ménage), comme le suggèrent les travaux sur l'individualisation des comportements sociaux, ou dans le cadre de réseaux sociaux plus larges.

Au-delà des résultats qui concluent à une forte contribution des migrants internationaux à l'économie domestique mais à leur influence beaucoup moins décisive sur les transformations de l'habitat des familles ${ }^{5}$, c'est à mon sens le concept de «ménage avec migrant» qui peut être discuté.

En effet, des travaux déjà anciens (en particulier ceux de l'équipe dirigée par Émile Lebris dans les années 1980) ont souligné les limites à considérer que "l'unité d'enquête, la famille, coïncide avec une résidence unique en ville ». Annick Osmont parle d'un système résidentiel familial articulant des lieux de résidence éclatés entre lesquels les liens de parenté sont fortement structurés (cérémonies familiales, mariages contrôlés, accueil des enfants dans le cadre de leur scolarité, etc.). Elle identifie dans ses travaux un «système» qui peut se déployer spatialement bien au-delà de la grande concession familiale ici entre Dakar, Rufisque, Kaolack et même Paris et Chartres. Le système résidentiel familial ainsi défini apparait plus opérationnel dans le contexte sénégalais que celui du "ménage» comme cadre d'observation de la circulation.

Émile Lebris rappelle également à la même époque que le changement de résidence «ne peut pas être le discriminant principal ou même exclusif de la migration au risque d'être totalement inadapté aux situations concrètes observées notamment en milieu urbain africain [...]. Le lieu ne suffit pas à situer l'individu; il y a donc nécessité à dépasser le contenu géographique de la notion de résidence et de considérer son contenu social. Sachant par ailleurs qu'il n'est pas évident de relier un individu aux différents groupes auxquels il appartient -

5http://www.ined.fr/fichier/t telechargement/57232 /telechargement fichier fr wp11 lessault etal 2010. pdf 
lignage, famille notamment - si on réutilise les définitions a priori de ces groupes ».

Si la catégorie de «ménage avec migrant » présente l'intérêt de dépasser les limites d'une restriction du ménage aux seuls occupants du logement, elle reste encore imparfaite pour rendre compte des circulations qui se réalisent au sein d'un système résidentiel familial multi-localisé. Elle constitue néanmoins une tentative de rattachement de la circulation internationale à un groupe de référence cohérent et opérationnel dans le cadre d'une enquête de nature quantitative. Elle appelle également un dialogue avec les procédés et concepts utilisés dans d'autres enquêtes afin de mieux caractériser le système-cadre des circulations.

David Lessault CR CNRS Migrinter - UMR 7301 Associé à l'Ined - UR 06 « Mobilité, logement et entourage » david.lessault@univ-poitiers.fr

\section{Bibliographie}

Le Bris, Émile; Marie, Alain; Osmont, Annik ; Sinou, Alain (1987) Famille et résidence dans les villes africaines. Dakar, Bamako, Saint Louis, Lomé, Paris, L'Harmattan, 268 p. (Villes et entreprises, 18).

Pilon, Marc ; Vignikin, Kokou (2007) Ménages et familles en Afrique subsabarienne, Paris, Ed. des Archives contemporaines, $122 \mathrm{p}$. (Savoirs francophones). 